\title{
The Ideal Implant Design from a Biomechanical Viewpoint
}

\author{
Biomechanics, Department of Polymeric Materials, Chalmers University of Technology, \\ Department of Research and Development, Astra Tech AB \\ Dr. Stig Hansson
}

A dental implant should be given a design which maximizes the anchorage strength and preserves the bone. Wolff's law implies a connection between mechanical events (stresses, strains) and biological events (bone modelling and remodelling) in bone. Its applicability on bone implants has been the subject for much controversy. However, a large number of animal and clinical studies testify to its applicability on dental implants. As an example the marginal bone resorption, often observed at implants with a smooth endosseous neck portion, can be understood in terms of Wolff's law. Wolff's law implies that an implant should be given such a design that high stress peaks in the bone are avoided and the functional loads induce a physiologic mechanical stimulation to all surrounding bone.

The above principles make it possible to evaluate different implant designs by means of advanced mathematical tools such as the finite element method. Below the results of a number of studies applying this paradigm are given.

If the endosseous neck portion of an implant is provided with retention elements as compared to having a smooth neck the peak bone stress resulting from an axial load is reduced most significantly. This implies a beneficial mechanical stimulation of the marginal bone in combination with an increase in the load that the implant can support.

The design of the implant-abutment interface affects the magnitude and the location of the peak bone stress resulting from an axial load. A conventional butt joint interface at the level of the crestal bone gives rise to a high peak stress where the implant starts to get attached to the bone. A conical implant-abutment interface at the same level results in a substantially reduced peak stress. Furthermore the peak stress lands up further down in the bone. This design appears to be the solution to a problem formulated by Mailath et al. 1989 ; to get the peak bone stress resulting from an axial load component spatially separated from the peak bone stress resulting from a horizontal load component. If the conical interface is moved $2 \mathrm{~mm}$ or more coronally, as in a one-component implant, these benefits of the conical design disappear.

For screw-shaped implants the profile of the thread affects the magnitude of the peak bone stresses. A micro-thread of a favourable design can be as effective as threads of normal dimensions.

The bone-implant interface shear strength can be increased by means of a rough surface. Conventional surface roughness parameters are quite inefficient when it comes to discriminate between surfaces giving good retention and surfaces giving poor retention in bone. A mathematical method was developed by means of which the efficacy of a rough surface can be estimated. According to this method a good surface roughness can be characterized as consisting of pits of a certain size and a favourable shape which are very densely packed. 